\title{
Some remarks on the genesis of scalar-tensor theories
}

\author{
Hubert Goenner
}

Received: 16 April 2012 / Accepted: 2 May 2012 / Published online: 7 June 2012

(C) The Author(s) 2012. This article is published with open access at Springerlink.com

\begin{abstract}
Between 1941 and 1962, scalar-tensor theories of gravitation were suggested four times by different scientists in four different countries. The earliest originator, the Swiss mathematician W. Scherrer, was virtually unknown until now whereas the chronologically latest pair gave their names to a multitude of publications on Brans-Dicke theory. P. Jordan, one of the pioneers of quantum mechanics and quantum field theory, and Y. Thiry, known by his book on celestial mechanics, a student of the mathematician Lichnerowicz, complete the quartet. Diverse motivations for and conceptual interpretations of their theories will be discussed as well as relations among them. Also, external factors like language, citation habits, or closeness to the mainstream are considered. It will become clear why Brans-Dicke theory, although structurally a déjà-vu, superseded all the other approaches.
\end{abstract}

Keywords Cosmology $\cdot$ Alternative theories of gravitation · History of Physics

\section{Introduction}

Among alternative theories of gravitation, scalar-tensor theories have received more attention than others. In publications using the English language, they are usually narrowed to a particular theory bearing the name Brans-Dicke theory, or sometimes, Brans-Dicke-Jordan theory. In the German physics literature, a then current name was Jordan's theory or "extended theory of gravity", in the French Jordan-Thiry theory. In the following conceptual description and interpretation of the genesis of scalar-tensor theories, we concentrate on the differing physical and mathematical motivations of the

H. Goenner $(\varangle)$

Institute for Theoretical Physics, University of Göttingen, Friedrich-Hund-Platz 1,

37077 Göttingen, Germany

e-mail: goenner@theorie.physik.uni-goettingen.de 
proponents of the theory, and on some factors outside of physics influencing the course of affairs (communications, distance to mainstream research, language skills, citation habits). It is interesting that all four scientists who suggested a scalar-tensor theory started from diverse vantage points and gave the scalar field differing interpretations. A methodical divide between these proposals is the choice between a projection from five-dimensional space and restriction to four-dimensional space-time from the outset. My special interest is to find out why the approach by Brans and Dicke succeeded to become a synonym for scalar-tensor theories (cf. [1]). If Nordström's relativistic scalar theory of 1913 is put aside [2], the nascency of scalar-tensor theories of gravitation has not been studied in any detail until now $[3,4]$. This includes the report given by C. Brans himself $[5,6]$.

There is a surprisingly large span of time concerning the publication of the four proposals: from 1941 to 1961 . One should think that at the beginning of the 1960s, i. e., about 20 years after the first proposal (Scherrer), and more than 10 years since the next two initiators (Jordan, Thiry) had made their work public, the main previous approaches to scalar-tensor theory would have been registered in the community of relativists. In Princeton, seemingly only Jordan's theory had become known, not Thiry's investigations in Paris, although these two groups had been citing each other. Moreover, chronologically, the main reference in the Princeton publications was to the second edition of Jordan's book published in 1955, with further references to papers of his group of the same and later years. But the essential results (and even more general ones than were reached by Brans-Dicke theory) had been published already until 1952, when the first edition of Jordan's book had come out. A conjecture would be that the interruption of communications during and right after the second world war has played a role. The unavailability of the first edition and of some of the less known German-language journals in which Jordan and his group in Hamburg published, may have hindered the spread of knowledge about his scalar-tensor theory. Also, the current citations habits in the physics community focusing more on recentness than on completeness of the literature referred to may have contributed. Moreover, contrary to the belief of some historians of science, i.e., that communication among scientists functions rapidly independently of the language used, it seems plausible that missing language skills, in this case mainly of French, are to be taken into account.

\section{From unified field theory to alternative gravitation}

\subsection{Jordan and projective relativity}

Towards the end of world war II, Pascual Jordan (1902-1980), one of the pioneers of quantum mechanics, had thought about processes by which stars are generated and about the time scale of their subsequent evolution. This seems to have played a major role for the building of a theory by him in which the gravitational constant $\kappa=\frac{8 \pi G}{c^{2}}$ is thought to be varying in (cosmological) time and is replaced by a scalar function. His starting point was Kaluza's unified field theory in a 5-dimensional space which formally had been rewritten as projective relativity in space-time (Veblen, Pauli). Its fifteenth field variable, a scalar which had been set constant, now was seen to nicely 
fit to Dirac's "large number hypothesis", i. e., his idea that the fundamental constants (including the age of the universe) might be variable in cosmological time [7,8].

In his first publication right after the end of the second world war, ${ }^{1}$ Jordan showed that Kaluza's field equations can be simplified by the introduction of five homogeneous coordinates. Their (homogeneous) transformations are isomorphic to the combined coordinate and gauge transformations. No mention of his later scalar-tensor theory is made in this note [9]. This was made up in 1946 in a brief note in which the fifteenth field variable of Kaluza's theory was identified by Jordan with the function to replace the gravitational constant $\kappa$ [10]. In projective relativity with projective coordinates $X^{\alpha}$, this is expressed by by setting $J:=g_{\alpha \beta} X^{\alpha} X^{\beta}(\alpha, \beta=0,1, \ldots, 4$ with $x^{0}$ being the time coordinate) equal to $\frac{2 \kappa}{c^{2}}$ [11]. In the article that followed, $\mathrm{G}$. Ludwig (1918-2007) displayed the relationship between variational principles in ${ }^{(5)} R$ and ${ }^{(4)} R$ [12]. The field equations for the gravitational field $g_{i j}$, the electromagnetic 4-potential $A_{k}=g_{4 k}$, and the $g_{44}$-variable $\kappa$ in space-time were then given by Jordan and Müller ${ }^{2}$ to be:

$$
\begin{aligned}
& G_{i k}+\frac{\kappa}{c^{2}} F_{i}^{s} F_{k s}=-\frac{1}{2 \kappa}\left(\nabla_{k} \nabla_{i} \kappa-\frac{1}{2 \kappa} \nabla_{i} \kappa \nabla_{k} \kappa\right), \\
& \kappa \nabla_{s} F^{s j}=-\frac{3}{2} \nabla_{s} \kappa F^{s j}, \\
& G=-\frac{\kappa}{2 c^{2}} F_{r s} F^{r s}+\frac{1}{2 \kappa} g^{r s} \nabla_{r} \kappa \nabla_{s} \kappa-\frac{1}{\kappa} g^{r s} \nabla_{r} \nabla_{s} \kappa .
\end{aligned}
$$

In January 1948, P. G. Bergmann (1915-2002) reported that work on a theory with a fifteenth field variable had been going on in Princeton:

"Professor Einstein and the present author had worked on that same idea several years earlier, but had finally rejected it and not published the abortive event" [13, p. 255].

It may be that, at the time, they just did not have an idea for a physical interpretation like the one suggested by P. Jordan. Although there were reasons for studying the theory further, Bergmann pointed out that there is an unwanted abundance in the theory: too many constructive possibilities for a Lagrangian. ${ }^{3}$ Nonetheless, in his subsequent paper on "five-dimensional cosmology", P. Jordan first stuck to the simplest Lagrangian, i.e. to the Ricci scalar in five dimensions [14]:

$$
{ }^{(5)} R={ }^{(4)} R+\frac{1}{4} J F_{r s} F^{r s}+\frac{2}{\sqrt{J} \sqrt{-g}} \partial_{r}\left(\sqrt{-g} g^{r s} \frac{\partial \sqrt{J}}{\partial x_{s}}\right) \text {. }
$$

\footnotetext{
1 Due to the warfare afflicting Germany, Europe and the whole world at the time, Jordan's first paper on the subject, submitted to Zeitschrift für Physik $\mathbf{4 6}$ in 1944, has not appeared. Jordan referred to proof sheets which I have not seen.

2 Contrary to common usage, Jordan and Müller denoted the Ricci tensor in space-time by $G_{i k}$. The covariant derivative refers to $g_{i j}$. Equations (11) and (12) of [11] corresponding to (2) and (3) here contain each a misprint. Both were corrected in [14].

3 Actually, this was known by Jordan and his collaborators, cf. below.
} 
2.2 Conceptional and formal developments

However, his co-workers Günther Ludwig and Claus Müller in 1948 generalized the Lagrangian to [15]:

$$
J\left[{ }^{(4)} R-\frac{J}{2} F_{r s} F^{r s}-\left(\lambda+\frac{1}{2}\right) \frac{\nabla_{r} J \nabla^{r} J}{J^{2}}\right] .
$$

Jordan had sent the proof sheets of this paper to W. Pauli already on 14 December 1947. Pauli pointed out that "your theory is completely equivalent with Kaluza's, if there is set $\frac{\partial}{\partial x^{5}}=0$, but $g_{55}$ is left arbitrary." He also found that "the additional term makes the theory less inevitable" [16, p. 510]. Already in 1952, in the first edition of his book, Jordan acknowledged the work of Ludwig and Müller and exchanged ${ }^{(5)} R$ for the expression:

$$
\kappa^{\eta}\left[{ }^{(4)} R-\zeta \frac{\nabla_{r} \kappa \nabla^{r} \kappa}{\kappa^{2}}-\frac{\kappa}{2 c^{2}} F_{r s} F^{r s}\right],
$$

where $F_{i j}$ is the electromagnetic field, $\eta, \zeta\left(=\lambda+\frac{1}{2}\right)$ dimensionless constants, and $J$ was replaced by $\kappa$. Ludwig and Müller had taken $\eta=1 .{ }^{4}$ In fact, in a further paper, Ludwig and Müller had included the matter-Lagrangian for a perfect fluid and obtained particular exact homogeneous and isotropic solutions for the equations of state $p=0$ and $p=1 / 3 \rho^{5}[15,17]$. One year later, Ludwig considered the general Lagrangian with three free functions $U, V, W$ :

$$
\mathcal{L}=\sqrt{-g}\left[U(J)\left({ }^{(4)} R+W(J) J_{, r} J_{, s} g^{r s}\right)+V(J)\right](r, s=1,2, \ldots, 4)
$$

and derived the corresponding field equations [18, pp. 550, 552, eq. (33)]. About 20 years later, Bergmann [19], and Wagoner [20] presented the same Lagrangian ${ }^{6}$ (7); the subsequent theory runs under the name "Bergmann-Wagoner theory" [21, pp. 123, 125]. Ludwig's paper is not referred to: both authors seemingly were unaware of its existence.

By going from $g^{i j}$ to $\psi^{-2}(\phi) g^{i j}$ and replacing $g_{i j}$ by $h(\phi) g_{i j}$, the following form of the Lagrangian with only two free functions was reached by Wagoner:

$\mathcal{L}=\sqrt{-g}\left[{ }^{(4)} R-n g^{r s} \frac{\partial \phi}{\partial x^{r}} \frac{\partial \phi}{\partial x^{s}}+2 \lambda(\phi)\right]+\mathcal{L}_{\text {matter }}\left(\psi^{2} g_{i j}, \ldots\right), \quad(n= \pm 1)$

\footnotetext{
4 In the 1 st edition of his book, Jordan not only gave (5) and (6), but also $J^{\alpha}\left[{ }^{(4)} R-\lambda \frac{\nabla_{r} J \nabla^{r} J}{J^{2}}\right]$ with numerical parameters $\alpha, \lambda$.

$5 p$ is the fluid's pressure and $\rho$ its density.

6 With functions $h(\phi), l(\phi), \lambda(\phi)$ in Wagoner's paper replacing $\mathrm{U}, \mathrm{W}$, and V. Bergmann had four free functions $f_{i}(\phi),(i=1,2,3)$ and $M(\phi)$ because he introduced the Maxwell Lagrangian separately instead of the matter Lagrangian.
} 
Ludwig seemingly had not been satisfied with the general Lagrangian (7): it had not contained a matter term. So, in his book, he presented the Lagrangian density as:

$$
\begin{aligned}
& J^{1 / 2} U(J)\left[\left({ }^{(4)} R+\frac{1}{2} J F_{r s} F^{r s}+J^{-1} g^{r s} \nabla_{r} \nabla_{s} J\right)\right. \\
& \left.+\left(W(J)-\frac{1}{2} J^{-2}\right) g^{r s} \nabla_{r} J \nabla_{s} J+V(J)+U^{-1} L_{\text {matter }}\right]
\end{aligned}
$$

Although for some time Jordan still clung to 5-dimensional theory, in their papers and in the book summarizing them he and his coworkers severed the "extended theory of gravitation" from Kaluza's theory and from the "unitary field theory" — approach of the French group around A. Lichnerowicz and Y. Thiry. G. Ludwig, C. Müller and K. Just continued their research by treating the theory as a theory alternative to general relativity. The period August 1954 to February 1956 was most fruitful for K. Just ${ }^{7}$ : he published 10 papers on Jordan's gravitational theory plus one with G. Ludwig. Apart from discussing possible Lagrangians and field equations for the theory, he applied it to relevant problems like planetary motion, cosmology, the torsion balance of Eötvös, and to more specific questions as were the Hertzian dipole or superpotentials. Aware of the ambiguities in the choice of the Lagrangian clearly shown by the different papers of the group, he integrated them into the expression for the Lagrangian:

$$
\kappa^{c(4)} R-s \kappa^{c-2} \partial_{r} \kappa \partial_{s} \kappa g^{r s}+\kappa^{c+1} L_{\text {matter }},
$$

where $c$ replaced Jordan's parameter $\eta$ [22]. Jordan had $c=1, s=\zeta[23$, p. 140, eq. (7)], K. Just had suggested $c=0$ [24] but in the same year 1955 Ludwig and Just had also looked at $c=-1, s=3 / 2$ [25, pp. 474/75]. In his application to the planetary system, more precisely to perihelion motion, Just started from (10) [26]. Just's proliferating publications probably did not help the cause of Jordan's theory: it was prone to baffle readers. Jordan could take notice of only one of Just's papers while proofreading the 2 nd edition of his book [27]. Just's Habilitationsschrift which gives a good overview of his contributions and a critical assessment of Jordan's theory apparently was not widely distributed. In it the value of $c$ in (10) is narrowed to [28, p. 161]:

$$
-2<c \leq-\frac{1}{2}, \quad c=0, \quad \frac{1}{2} \leq c \leq 2
$$

\subsection{Mass generation in the universe?}

In the 1 st edition of his book, as a consequence of the field equations, Jordan had obtained the equation $\left(\kappa^{2} T^{\alpha \beta}\right)_{; \beta}=0$ and interpreted it as invalidating conservation of matter "in the old style, although, as required in principle by relativity, a definite form of conservation law must still exist" [23, p. 143]. A certain reservation is

\footnotetext{
7 Kurt Just, Professor Emeritus of Physics, University of Arizona, Tucson.
} 
expressed by him when he wrote on the same page: "Therefore, we reserve the right to accept the theoretical results only in part if the above equations are applied to processes of matter generation." Nevertheless, he took it seriously: Applied to a single star this meant that "the single mass of a star $M_{S t}$ must have the property that its multiplication with $\kappa^{2}$ must yield something constant: $\kappa^{2} M_{S t}=$ const." (ibid., p. 183). Also in his letter to Pauli of 9 June 1953, Jordan defended his view, not without a portion of imaginativeness: "[...] The calculation of the augmentation or generation of mass supposedly is only of statistical importance; real generation of mass probably does not happen at the places where star-masses exist but obtains abruptly with the generation of new stars, by fusion of our cosmos with 'embryonic stars'" [29, p. 178]. ${ }^{8}$

In the second edition, following Eddington and Pauli, another interpretation was introduced: "What is conserved, we call mass or energy" [22, p. 171]. Consequently, the matter tensor is not $T^{\alpha \beta}$ but $\kappa^{2} T^{\alpha \beta}$. This was criticised in a book review by $\mathrm{O}$. Heckmann who argued that then the meaning of $\kappa$ as gravitational coupling function again is questionable. He also found Jordan's "cosmogonic" consideration very speculative: "he abandons the ancient [...] assumption of the conservation of matter" [30, p. 282].

\subsection{Criticism by Pauli and Fierz}

Jordan's theory received wider attention after his and G. Ludwig's books had been published in the early 1950s [23,31]. In a letter to Jordan, Pauli criticized projective relativity as bringing no progress with regard to Kaluza's theory and questioned Jordan's taking the five-dimensional curvature scalar ${ }^{(5)} R$ as his Lagrangian.

One of those responding also to this book was M. Fierz in Basel. Previous to the publication of his paper [32], he had corresponded with W. Pauli. In following an idea of Lichnerowicz [33, p. 201], he interpreted the scalar function as "permittivity of the vacuum" (the electric constant) $\varepsilon_{0}=1 / \mu_{0}=\kappa^{2}$ and pointed to a difficulty of coupling the scalar field to the energy-momentum tensor of matter. In fact, for the transition from the gravitational constant $\kappa$ to a scalar field function $\phi$, it makes a difference whether the Einstein Lagrangian is written as ${ }^{(4)} R+\kappa L_{\text {matter }}$ or as $\kappa^{-1(4)} R+L_{\text {matter }}$. This is one of the differences between Jordan's and Brans-Dicke scalar-tensor theory. Fierz suggested to introduce either point particles as matter or a (quantum-) Klein-Gordon field in order to remove conformal invariance. He sent the first versions of his paper to Pauli and eventually received Pauli's placet; cf. the letter of W. P. to M. F. of 2 June 1956 in [34, p. 578].

In the 2 nd edition of his book, Jordan then discussed a difficulty of his theory pointed out by W. Pauli in this context: instead of $g_{i k}$ equally well $\phi(x) g_{i k}$ with arbitrary function $\phi$ could serve as a metric. Jordan's theory is conformally invariant only in the case that an electromagnetic field forms the matter tensor (particles with zero rest-mass). A problem for the interpretation of mathematical objects as physical variables results: by

\footnotetext{
8 "Die dann zu berechnende Massenvermehrung oder Massenerzeugung geht wohl nicht an den Stellen vorhandener Sternmassen vor sich, sondern erfolgt durch schlagartige Erzeugung neuer Sterne durch Verschmelzung unseres Kosmos mit 'embryonalen Sternen.',"
} 
a suitable choice of the conformal factor $\phi$, a "constant" gravitational coupling function could be reached, again. In the 2 nd edition of his book, Jordan introduced a new section on "Pauli's conform-transformations" [22, §28]. Fierz's interests in Jordan's theory was not strong, but mainly of pedagogical nature. In this context, in a letter to N. Straumann, Zürich, he wrote [35]: "Die Absicht meiner Publikation war also vor allem pädagogisch." 9 Both, Pauli and Fierz gave a low rating to Jordan's theory. ${ }^{10}$ In 1958, Pauli renewed his criticism concerning the five-dimensional curvature scalar ${ }^{(5)} R$ as the Lagrangian of Jordan [36, note 23, p. 230].

In 1959, Jordan had fully accepted the criticism by Pauli and Fierz:

"If one goes along with the considerations communicated by Fierz-and it seems to me that they are imperative-then apparently Dirac's hypotheses II can no longer be upheld, because [...] none of the field equations derivable from a variational principle [...] permits a violation of mass conservation." 11

As to the special choice of $c(\eta)=-1$ in Eq. (10), his argument was that otherwise, as had been noted before by Lichnerowicz for $c(\eta)=+1$, the permittivity of the vacuum $\varepsilon_{0}=\kappa^{1+\frac{1}{\eta}}$ would depend on $\kappa$.

\section{Scherrer and elementary particles}

Neither Pauli nor Fierz, lecturer in Basel, Switzerland, at the time, payed attention to the fact that a mathematician at the university of Bern had suggested a scalar-tensor theory already in 1941 before P. Jordan, and without alluding to Kaluza's theory or to Pauli's projective formulation. The short note, written in German, was not readily accessible outside the German speaking countries and carried the title "Zur Theorie der Elementarteilchen" (About the theory of elementary particles) [38]. In this note, the mathematician Willy Scherrer (1894-1979) of Bern, Switzerland, introduced a scalar field $T\left(x^{0}, x^{1}, x^{2}, x^{3}\right)$ and a variational principle

$$
\delta \int T R \sqrt{-g} d^{4} x=0
$$

\footnotetext{
9 And in a footnote in the letter:

"Dies gilt für alle meiner Arbeiten. Ich habe nie etwas erfunden oder entdeckt. Aber ich konnte erklären, was die Formeln physikalisch bedeuten: Jordan zeigt, wie man es nicht machen soll" (This holds for all of my papers. I never invented or discovered something. But I could explain the physical meaning of the formulas: Jordan is showing how things should not be done.).

10 See letters of W. P. to M. F. of 30. 9. 1955, p. 350 and 2. 3. 1956, p. 531; of M. F. to W. P. of 8/11. 3. 1956, p. 539 in [34].

11 Schließt man sich den von Fierz mitgeteilten Erwägungen an—und es scheint mir, daß sie tatsächlich zwingend sind - so scheint es zunächst, daß die Diracsche Hypothese II nicht mehr vertretbar ist, da weder $[\ldots]$ noch irgendwelche aus einem Variationsprinzip ableitbaren Feldgleichungen [...] eine Durchbrechung der Massenerhaltung zulassen [37].
} 
with the constraint

$$
\int T \sqrt{-g} d^{4} x=\text { constant }
$$

where $R$ is the curvature scalar of space-time. The constraint reminds us of the norming of a Schrödinger wave function $(T \sim \psi \bar{\psi})$ and, in fact, Scherrer gave as his motivation "new Ansätze for a scalar relativistic wave mechanics." 12 In the same year, Scherrer had tried to describe the interaction of two particles by means of, as he said, "relativistic Schrödinger equations" [39,40]. He finally had come up with a system of two coupled relativistic wave equations for two wave functions $u, v$. Interestingly, in his correspondence with W. Pauli in connection with a discussion of Dirac's equation in his extended theory of gravitation, Jordan claimed that $\int \kappa^{2} \psi \psi^{*}$ is time-independent. Pauli agreed and gave as the commutation relations of "second quantization": $\kappa^{2}\left\{\psi_{\alpha}(x, t), \psi_{\beta}^{*}\left(x^{\prime}, t\right)\right\}_{+}=\delta_{\alpha \beta} \delta^{(3)}\left(x-x^{\prime}\right)$. But he added "[ [...] everything goes as if $\Psi=\kappa \psi$ is introduced as a new spinor and $\kappa$ is omitted" [29, pp. 178, 180, 194].

In his first note, Scherrer proposed a generalization of his Lagrangian in (12) to $T\left[R+k(\operatorname{grad} \ln T)^{2}\right]$ with a constant $k$ and $(\operatorname{grad} \ln T)^{2}$ obviously abbreviating $\frac{k}{T^{2}} g^{r s} \frac{\partial T}{\partial x^{r}} \frac{\partial T}{\partial x^{s}}$. This corresponds to Jordan's theory.

Six years later, Scherrer had seen a paper by Ludwig and Müller [17] and probably hastened to secure priority for himself by elaborating on his previous note. He now presented another argument for the replacement of Einstein's equations [41, p. 537]: "In the case of vanishing matter, one should expect degeneracy of the metrical structure from a theory which makes matter responsible not just for deviations from geodesic motion but for the total metrical structure." 13 As a cure for this apparent deficiency, he suggested to allocate to each place in the world an "intensity" $\psi^{2}$ such that $\psi^{2} \sqrt{-g} d^{4} x$ stands for the relative number of material elements lying in the volume element $\sqrt{-g} d^{4} x$. Thence, he interpreted his theory as an alternative to Einstein's theory.

In place of the manifest ${ }^{(4)} R \psi^{2} \sqrt{-g} d^{4} x$, Scherrer advocated the more general Lagrangian of his note of 1941 [38, p. 238]:

$$
\mathcal{L}=\left[\left({ }^{(4)} R-2 \Lambda\right) \psi^{2}+4 \omega g^{r s} \frac{\partial \psi}{\partial x^{r}} \frac{\partial \psi}{\partial x^{s}}\right] \sqrt{-g}
$$

$\psi$ corresponds to a scalar matter field. ${ }^{14}$ In the paper, he showed that for $\Lambda=$ $\omega=0$ a static and spherically symmetric exact 2-parameter solution existed containing the Schwarzschild solution as a special case. He calculated its "total energy" $\int T_{0}^{0} \sqrt{-g} d^{4} x$ to be finite. The model-system being in "complete equilibrium"

\footnotetext{
12 "Veranlasst durch neue Ansätze zu einer skalaren relativistischen Wellenmechanik [...]."

13 "Von einer Theorie, die die Materie nicht nur für die Abweichungen von der Trägheitsbahn, sondern für die totale metrische Struktur verantwortlich macht, sollte man eigentlich erwarten, dass sie im Falle verschwindender Materie entartet."

14 Scherrer had also thought about a vector field as matter, but discarded the idea. Cf. [41, p. 539].
} 
according to Scherrer, "it logically first should be applied to elementary particles." 15 He gave two reasons for why quantization had not yet occurred in his theory: (1) The restriction to a static situation might be too restrictive and, (2) quantization possibly might first show up in a 2-body-problem. In a sequel to this paper, Scherrer then considered the case $\Lambda=0, \omega \neq 0$ and again calculated the solution corresponding to the Schwarzschild solution in General Relativity [42]. He submitted exact solutions for the static, spherically symmetric case in 195010 days later than Heckmann, Jordan and Fricke $(\eta=1)$ did for Jordan's theory [43].

Scherrer advised a doctoral student, K. Fink, to continue his approach. But Fink looked at the Lagrangian density $\mathcal{L}=\left(R+2 \omega g^{r s} \frac{\partial \psi}{\partial x^{r}} \frac{\partial \psi}{\partial x^{s}}\right) \sqrt{-g}$ [44]. In it the coupling of the scalar matter field $\psi$ to the curvature scalar is missing! The ensuing field equations correspond to Einstein's equations for a massless scalar field or, equivalently to those following from Jordan's Lagrangian if the parameter $\eta=0$ [23, p. 140]. Fink obtained static, spherically symmetric solutions as well as solutions for a homogeneous and isotropic cosmological model.

\section{Thiry and mathematical physics}

In January 1950, Yves Thiry submitted a thesis to the faculty of science of Paris University with the title "Mathematical study of the equations of a unitary theory with fifteen field variables". He exuberantly thanked his "master and friend Lichnerowicz" who obviously had initiated the work. Unfortunately, "Jordan and his school" had "obtained almost at the same time like us the equations which we will give in Chapter II. We had no knowledge about this except at a very late stage, and it is only recently that we could correspond with Jordan. He was so friendly as to send us his publications which we could not have procured otherwise" $\left[45\right.$, p. 6]. ${ }^{16}$ In fact, it was A. Lichnerowicz (1915-1998), then at the University of Strasbourg, who had written to W. Pauli and asked for "Jordan's original paper" (cf. letter of W. Pauli to P. Jordan of 23. 3. 1948 in [16, p. 516]). According to Pauli:

"Lichnerowicz is a pure mathematician who is occupied with the integration of

Einstein's field equations. One of his students, Ives Thiry now has looked into the (not mutilated) Kaluza-theory (with $g_{55}$ ) and, so I believe, has simplified very much the calculational technique."17

Since 1947/1948, first together with A. Lichnerowicz, Thiry had published short notes about Kaluza's theory in Comptes Rendus. In the first note of February 1947 on variational calculus, after presenting a certain mathematical formula they stated:

\footnotetext{
15 “[...] muss es sinngemäss in erster Linie für Elementarteilchen in Aussicht genommen werden.”

16 “[...] obtenu à peu près en même temps que nous les équations que nous donnons au Chapitre II. Nous n'avons eu connaissance de ce fait que fort tard et ce n'est que récemment que nous avons pu correspondre avec Jordan, qui a eu l'amabilité de nous envoyer ses publications qu'il était alors impossible de se procurer autrement."

17 "Lichnerowicz ist ein reiner Mathematiker, der sich mit der Integration der Einsteinschen Feldgleichungen befasst. Einer seiner Schüler, Ives Thiry hat sich nun mit der (unverstümmelten) Kaluza-Theorie (mit $g_{55}$ ) beschäftigt und, glaube ich, die Rechentechnik sehr vereinfacht."
} 
“Therefore, Kaluza's theory presents itself as an immediate application of formula (5) which even may lead to an extension of this theory to a theory with $g_{00} \neq 0$ " [46, p. 531]. The 15 field equations then were given by Thiry in January 1948 [47]. ${ }^{18}$ On 19 January 1948, ${ }^{19}$ he sent this second note in Comptes Rendus to Albert Einstein [47]. In his publication preceding Lichnerowicz' letter to Pauli, Thiry had not yet given a physical interpretation of the scalar field [47]. Around that time, the interest in 5-dimensional relativity seems to have risen; we already have met P. G. Bergmann's paper of 1948 [13]. ${ }^{20}$ C. V. Jonsson, a student of O. Klein in Stockholm, also wrote a long paper about the theory's field equations. He included the scalar field and dropped the cylinder condition. ${ }^{21} \mathrm{He}$ then quantized the free field in linear approximation [49]. Thiry's interest in Kaluza's theory was of mathematical nature:

"As to unitary field theories, it seems that their mathematical study has been quite neglected [...]. We thought it useful to try a systematic mathematical study of a unitary field theory, and to find out whether such a theory is able to present the same coherence like general relativity." [45, p. 3] ${ }^{22}$

In three chapters, Thiry's thesis laid out the conceptual background of a 5-dimensional theory, the setting up and study of the field equations by help of Cartan's differential calculus, and results on regular solutions of the theory's field equations. Unlike in the approach by Einstein and Bergmann, the cylinder condition $g_{\alpha \beta, 4}=0$ is upheld, throughout. Here he used an argument from physics: no physical phenomena furnished evidence for the existence of a fifth dimension [45, p. 39]. His access to 5-dimensional space used the fact that the equations of motion of charged particles are geodesics in Finsler geometry; for each value of $\frac{e}{m}$ (charge over mass) another Finsler space is needed with the metric:

$$
d s=\sqrt{g_{j k} d x^{j} d x^{k}}+\frac{e}{m} A_{l} d x^{l} .
$$

He then showed that a 5-dimensional Riemannian space could house all these geodesics. $^{23}$

"The introduction of a fifth coordinate [...] thus shall justify itself by the fact that it imparts the role of geodesics to the trajectories of charged particles which they lost in space-time $[\ldots], 24$

\footnotetext{
18 In the third paper, the global problem whether regular solutions exist was dealt with [48].

19 The call number of the Einstein Collected Papers (ECP) is 16-312.00.

20 Bergmann's paper appeared only on January 1, 1948 although it had been submitted on August 30, 1946. Thus he could not yet have reacted to Thiry's correspondence with Einstein.

21 I have not been able to verify that Jonsson's field equations for the case of the cylinder condition agree with Thiry's equations.

22 "Quant aux theories unitaires, il semble que leur étude mathématique ait été relativement negligée [...] Il nous a paru utile de tenter une étude mathématique systématique d'une théorie unitaire et de voir si une telle théorie est susceptible de présenter la même cohérence que la théorie de la Relativité générale."

23 In nuce, this idea can already be found in his paper with Lichnerowicz [46, p. 531].

24 "L'introduction d'une cinquième coordonnée, $[\ldots]$ se justifiera donc par le fait qu'elle confière aux trajectories des particules électrisées le rôle de géodesiques qu'elles perdaient dans l'espace-temps [...]".
} 
In the third chapter, Thiry aimed at showing that his unitary field theory possessed the same mathematical coherence with regard to its global aspects. By partially using methods developed by Lichnerowicz, he proved theorems on the global regularity of solutions. These results are of a different nature than what Jordan had achieved; they are new and mathematically rigorous.

Yet Jordan was not in a hurry to read Thiry's thesis; he wrote to Pauli:

"By the way, in his thèse published in 1951, Thiry has studied systematically and extensively the theory with variable gravitational constant; [...] I received it only after my book appeared and, at present, I have not read it very closely. It thus is not really clear to me whether it contains interesting novelties." [50, pp. $799 / 800]^{25}$

To Pauli, Thiry's global theorems might not have been "interesting novelties", because in his corresponding paper with Einstein on the non-regularity of solutions, the proof had been independent of the dimension of space [51]. Pauli, at first, also did not read Thiry's thèse, but responded hard-nosed:

"The Thèses by Thiry are laying on my desk; however they are so appalingly thick (do not contain a reasonable abstract) such that it is so much simpler to not open the book and reflect about what must be inside." (W. Pauli to P. Jordan 8. 6. 1953, [29, p. 176] $)^{26}$

Somewhat later, Pauli corrected himself and wrote to Jordan that in the preparation for a course "he nevertheless had read around in Thiry's Thèse" (W. Pauli to P. Jordan 3. 2. 1954, [29, p. 442]). Note that neither of these two eminent theoretical physicists discussed Thiry's paper as regards its valuable content.

As to the field equations corresponding to Eqs. (1)-(3), Thiry had calculated them in great detail with Cartan's repère mobile for both a euclidean or Lorentz metric of $V_{5}$, and also with a 5-dimensional matter tensor of the form of dust $\rho u^{\alpha} u^{\beta}$. From the 15th equation, he even had obtained "a new physical effect": uncharged dust-matter could generate an electromagnetic field [45, p. 79, footnote (1)]. He linked this effect to Blackett's search for the magnetic field of a gravitating rotating body. Lichnerowicz and his doctoral student Francoise Hennequin discussed consequences of the projection of the matter tensor into space-time: a charged perfect fluid could be described. This included the interpretation of $\kappa=\frac{G}{\phi}$ as "a gravitational factor" in front of the matter tensor in space-time reducing to the gravitational constant for $\phi=1$ by Lichnerowicz [33, p. 202]. Thiry himself kept Jordan's original choice ${ }^{(5)} R$ for the Lagrangian.

\footnotetext{
25 "Thiry hat ja übrigens in seiner 1951 veröffentlichten Thèse die Theorie mit variabler Gravitationskonstante systematisch und ausführlich studiert;[...] Ich habe es erst nach dem Erscheinen meines Buches von ihm bekommen und augenblicklich noch nicht sehr genau gelesen. Ich weiß also auch noch nicht recht, ob interessante Neuigkeiten darin stehen."

26 "Die Thèses von Thiry liegen auf meinem Tisch; sie sind aber so entsetzlich dick (haben auch keine vernünftige Zusammenfassung), daß es so viel einfacher ist, das Buch nicht aufzumachen und sich zu überlegen, was darin stehen muss."-Thiry's thesis comprises 122 pages.
} 


\section{Princeton: R. Dicke and Mach's principle}

Since the mid 1950s, Robert H. Dicke (1916-1997) had been thinking about Dirac's large number hypothesis, the equivalence principle, and Mach's principle [52,53]. According to Sciama, an expression for this principle is given by $G V=-c^{2}$, where $\mathrm{V}$ is the gravitational potential of the universe and $G$ the gravitational constant. Qualitatively, thus $G M / R c^{2} \sim 1$, whith $M$ the mass of the observable universe, $R$ the radius of the boundary of the observable universe, and $c$ the vacuum velocity of light [54].

In another paper, Dicke may have taken up the idea of Fierz when he wrote: "that a space variation in the polarizability of the vacuum will lead to a number of results familiar as typical gravitational effects." In this context, he investigated "a form which a theory of gravitation may take when the principle of equivalence is satisfied in a weakened form only" [55, p. 363]. He went on: "Jordan has previously considered a similar problem and Fierz has made a critical analysis of Jordan's theory." References to Jordan's book (2nd edition 1955) and the paper of Fierz are given. ${ }^{27}$ The theory was to accept Mach's principle, the cosmological principle, and general covariance. A theory for a scalar field $\epsilon$ in a flat background is suggested having the Lagrangian: $L=\frac{1}{k} h^{i j} \epsilon_{, i} \epsilon, j$ with $h^{i j}$ being the reciprocal of the symmetric tensor $g_{i j}$ describing the vacuum with $g_{11}=g_{22}=g_{33}=-\epsilon$; $g_{44}=1 / \epsilon$. The quantity $\epsilon \rightarrow \varepsilon$ is taken as the electric specific inductive capacity of the vacuum. This looks rather different from the motivation of Scherrer or Jordan for their scalar-tensor theories theories. It turns out that, in Dicke's approach, asymptotically, the scalar field is proportional to the inverse of the ratio of the gravitational to the electromagnetic interaction between two elementary particles on an atomic scale. Thus, a connection to Dirac's hypothesis is established [55, p. 375].

What now is called Brans-Dicke scalar-tensor theory then was published in 1961 by C. H. Brans and R. H. Dicke [56] and in a subsequent paper by Brans [57]. In the joint paper, no reference was given to Brans' dissertation submitted to the Princeton faculty in May 1961 by Brans [58]. In the first publication, Dicke's previous paper of 1957 was also not mentioned. The authors were at pains to distance their theory from Jordan's: "There is a formal connection between this theory and that of Jordan, but there are differences and the physical interpretation is quite different" [56, p. 928]. As a second reference besides Jordan's book of 1955, his paper of 1959 [37] was cited with the comment: "In this second reference, Jordan has taken cognizance of the objections of Fierz $[\ldots]$ and has written his variational principle in a form which differs in only two respects from that expressed in Eq. (16)." ${ }^{28}$ The name "Brans-Dicke theory" was used by Dicke right away [59, p. 2167], [60, p. 656, caption of figure 1].

\footnotetext{
27 According to Dicke (p. 364): "The fact that many of the properties of gravitation can be accounted for in terms of an interaction with a polarizable medium is an old idea which has recurred from time to time." He then cited a paper of $\mathrm{H}$. A. Wilson of 1921.

28 The reference to Eq. (16) is a typo; Eq. (6) of [56] must be meant.
} 
The Lagrangian presented by Brans and Dicke is:

$$
\mathcal{L}=\left(R \phi-\frac{\omega}{\phi} g^{r s} \frac{\partial \phi}{\partial x^{r}} \frac{\partial \phi}{\partial x^{s}}\right) \sqrt{-g}
$$

This is exactly Scherrer's expression (14) published in 1941 and 1949 if $\phi=\psi^{2}$, the cosmological constant $\Lambda=0$, and Scherrer's constant $\omega$ is replaced by the constant $-\omega$ in the publication of Brans and Dicke. ${ }^{29}$ The field equations obtained from (14) are:

$R_{i j}-\frac{1}{2} g_{i j} R=\frac{8 \pi}{c^{4}} \phi^{-1} T_{i j}+\frac{\omega}{\phi^{2}}\left(\phi_{, i} \phi_{, j}-\frac{1}{2} g_{i j} \phi_{, r} \phi_{, s} g^{r s}\right)+\phi^{-1}\left(\phi_{, i ; j}-g_{i j} \square \phi\right)$.

This equation had been given already in 1948 by Ludwig and Müller [15,17]. The scalar wave equation following from (16) is:

$$
2 \omega \phi^{-1} \square \phi-\omega \phi^{-2} \phi^{, i} \phi_{, i}+R=0 ;
$$

it had already been derived in 1948 by Ludwig and Müller [15, 17].

Now, the advantage of the choice $\kappa=\phi^{-1}$ in Brans-Dicke theory is that the field equation for $\phi$, after manipulation of Eqs. (17), (18), becomes the wave equation:

$$
\square \phi=\frac{8 \pi T}{2 \omega+3},
$$

where $T$ is the trace of the matter tensor, and $\square \phi=g^{r s} \nabla_{r} \nabla_{s} \phi$ the covariant d'Alembertian. Sciama's expression for Machs principle is an immediate consequence in the static case. It is to be noted that Scherrer had ${ }^{30}$

$$
(3+2 \omega) \square \chi-2 \Lambda \chi=0 .
$$

The two differing points mentioned above were: (1) Jordan set $\kappa=\phi$, while Dicke and Brans chose $\kappa=\phi^{-1}$; (2) "[...] as a result of its outgrowth from his five-dimensional theory, Jordan has limited his matter variables to those of the electromagnetic field." In view of Jordan's discussion of the matter tensor in connection with its nonconservation, and the presentation of cosmological solutions for an ideal fluid in $\$ 30$ of his book [22, pp. 186-196], this second point looks rather contrived. The publication by Brans and Dicke was a first short summary of the dissertation of C. H. Brans. It included the weak-field equations, a static, spherically symmetric exact solution applied to the calculation of the perihelion shift and light deflection deviating from Einstein's theory by the factors $\frac{3+2 \omega}{4+2 \omega}$ and $\frac{3 \omega+4}{3 \omega+6}$, respectively. These values had been

\footnotetext{
29 However, the definitions of the curvature tensor by Scherrer and by Dicke-Brans differ by a minus sign. Thus, in the results the symbol $\omega$ stays the same. Cf. (20) below.

30 His eq. (2.19) on p. 542; cf. [41].
} 
calculated before by Heckmann et al. [43, pp. 139, 141]. ${ }^{31}$ Also, a derivation of Mach's principle in the form $G M / R c^{2} \sim 1$, and a discussion of homogeneous and isotropic cosmological models were given. The follow-up by Brans alone contained a thorough investigations of the relationship of a locally measured gravitational constant and "the structure of the universe" as well a discussion of the boundary conditions for the scalar field, its consequences for a spherically symmetric mass distribution, and conservation laws (cf. [57, sections II-IV]).

All this had been worked out in detail in Brans' dissertation. In two chapters of it (VI C, pp. 37-38 and VIII A-G, pp. 57-68), he discussed Jordan's work as presented in the 2nd edition of his book, in a later paper of 1959 [37], and also publications of K. Just [26,61,62] and G. Ludwig [25] from 1955 and 1956. In these papers, Jordan's theory had been applied to planetary motion, precession of the perihelion and to cosmology. Also, his theory of the generation of matter had been discussed. In his thesis, Brans made it very clear that one of the main incentives for Dicke's and his work was to keep the weak principle of equivalence and to leave unchanged "the entire theory of matter and electromagnetism" plus the "Coulomb determination of inertial mass used in general relativity" [58, p. 36]. However, Dicke's "strong" principle of equivalence was violated. ${ }^{32}$ The spherically symmetric vacuum solution of (17), (18) by Heckmann and Fricke, discussed in $\$ 29$ of Jordan's book was presented and compared to the exact solutions Brans had obtained himself in isotropic coordinates. Thus, while Brans carefully examined Jordan's theory as presented in and after 1955, he and Dicke were silent on the earlier papers of the Hamburg group and on the contributions of the Paris group. This may be due to their belief that it be unnecessary to cite earlier references already listed in the second edition of Jordan's book. Nevertheless, this made appear the work of Dicke and Brans prompter in time concerning the time-scale of publications. They rightly felt no need to cite the paper by Jonsson of 1951 [49], written in English and refered to in Jordan's book because Jonsson's paper dealt with 5-dimensional space and did not suggest another scalar-tensor theory.

\section{Stockholm's Högskola: Jonsson and Klein}

C. W. Jonsson's knowledge of the literature in the field in which he set out to work must have been limited: although starting from 5-dimensional theory, he did not mention Kaluza's name. The work was done under supervision by Oskar Klein (1894-1977) in Stockholm who, in the 1920s, had given Kaluza's theory a new physical interpretation by closing the fifth dimension: He took 5-dimensional space essentially as a four dimensional one with a small periodical strip or tube in the additional spacelike dimension affixed. The 4-dimensional metric then is periodic in the additional

\footnotetext{
31 For the parameters in both papers we have $\alpha_{0}=-\frac{1}{2+\omega}$.

32 Brans defined Dicke's strong equivalence principle: "as the assertion that in the absence of non-inertial and non-gravitational forces, the numerical content of experiments performed in a locally almost flat physical coordinate system is independent of any characteristics of the mass distribution in the rest of the universe" [58, p. 26].
} 
coordinate which Klein then related to Planck's constant [63,64]. ${ }^{33}$ In 1938 and 1946, O. Klein had tried to geometrize the meson field via the Kaluza-Klein 5-dimensional theory and allowed all field quantitied to be periodic functions in the additional coordinate $[66,67] .{ }^{34}$ In view of the fact that Jonsson derived the field equations without prescribing the cylinder condition, i.e., by allowing the components of the 5-dimensional metric to be dependent on the fifth coordinate, he also could have cited Einstein and Bergmann. In their new approach in 1938, they had claimed to ascribe "physical reality to the fifth dimension whereas in Kaluza's theory this fifth dimension was introduced only in order to obtain new components of the metric tensor representing the electromagnetic field" [70, p. 683]. That Klein advised a student to extend Kaluza's theory by allowing the additional fifth coordinate in all field variables, may have been a consequence of Pauli's violent reaction to Klein's talk given in Princeton in winter 1949/1950. Klein then had insisted on keeping the cylinder condition (cf. [50, p. XXV]).

Moreover, Jordan and his co-workers remained uncited. Certainly, Jordan's book had appeared only in 1952, but he and his collaborators had published papers before in Annalen der Physik and Zeitschrift für Naturforschung both of which might have been available in Stockholm at the time. ${ }^{35}$ Also, G. Ludwig's book had appeared in 1951, which contained "the results of some papers of P. Jordan, G. Müller and the author as well as some not yet published results" [31, p. 3]. Thiry's thesis was mentioned by Jonsson although it had just been published at the beginning of 1951. Jordan himself felt encouraged by Jonsson's paper. In it, as in Thiry's approach, the same extension of Kaluza's theory had been performed which he had suggested; hence he no longer could be seen as an outsider [22, p. 161]. There is a correspondence between Pauli and O. Klein about Jordan's theory only later in 1953 [29, p. 209].

Whatever the reasons for Jonssons negligent way of citing may have been, his motivations were quite different than those used in the other approaches described above: he was not interested in scalar-tensor theory as a theory of gravitation but in its field quantization in linear approximation. Hence he set $\gamma_{\mu \nu}=\delta_{\mu \nu}+\epsilon \phi_{\mu \nu}$ with $\left|\phi_{\mu \nu}\right| \ll 1 ;(\mu, v=0,1,2, \ldots, 4)$ The conditions $\frac{\partial \phi_{\mu 4}}{\partial x^{4}}=0$ now were added. ${ }^{36} \phi_{r s}$ with $r, s,=0,1,2,3$ was assumed to be periodic in $x^{4}$ and expanded in a Fourier series. With the scalar field set constant, the quantization of the free field of spin $2 \hbar$ was then carried through in Fock-space. C. V. Jonsson seems to have left no further traces in theoretical physics.

\footnotetext{
33 This has been acknowledged by Pauli in his re-edition with comments of his encyclopedia-article of 1921. Cf. [65, note 23, footnote 4 on p. 275].

$34 \mathrm{Cf}$. For O. Klein als a pioneer of gauge theory applied to the nuclear forces cf. [68, p. 32]. An English translation of [66] can be found in [69].

35 Sweden was a neutral country during the second world war such that the flow of scientific communication with Germany should not have been hampered; this includes the period directly after the war. On the contrary, according to W. Pauli, during the war it was impossible to send printed matter from the USA to Sweden.

$36 x^{4}$ is the fourth space-coordinate; $x^{0}$ denotes the time-coordinate.
} 


\section{Problems in communication}

In his personal memories, Carl Brans stated that during the writing of his thesis: "[...] I discovered the work of Jordan et al. on this topic and almost quit writing. However, I was encouraged to continue, giving what I hope is sufficient and appropriate credit to Jordan and his group" [6, p. 5]. He does not say how he discovered Jordan. In view of the references to Jordan's book given by his thesis adviser Dicke already in a publication of 1957 and in two of 1959 [52, p. 363], [53, p. 356], it seems that the discovery cannot have been of an abrupt nature. At the same time at which Brans wrote up his thesis, the Princeton postdoc Dieter Brill ${ }^{37}$ spent the year 1960/1961 in Hamburg at the Physikalisches Staatsinstitut as an independent Flick exchange fellow. There has been some contact between Brill and Dicke in the preparation of the Enrico Fermi Summer School of 1962, where both gave lectures with Brill reviewing Jordan's theory [71]. His detailed presentation of results by Jordan and some of his associates (among others from K. Just's Habilitationsschrift) was the first one for English speaking relativists. However, there has been no direct interaction between D. Brill and C. Brans concerning Jordan. ${ }^{38}$ It also is unlikely that there has been a correspondence between Brans' thesis adviser R. Dicke and P. Jordan as in the case of Lichnerowicz and Thiry.

D. Brill speaks German perfectly; Carl Brans also can read German. Moreover, Peter Bergmann who had not cited the paper of G. Ludwig was of German origin and thus could read the language fluently. In Princeton, Valentin Bargmann whom Dicke had thanked in his paper on a special relativistic treatment of gravity, although of Russian origin, had worked long enough with Wentzel, Pauli, Bergmann and Einstein to be proficient in German. Thus, with regard to the papers of Jordan and his associates, language problems should not have played a major role in Princeton. With French, it might have been different. Mme. Tonnelat's book on unified field theory of 1955 [72] was translated into English only in 1966. Lichnerowicz's book on relativistic theories of gravitation of the same year [33] in which his and Thiry's results were presented and Tonnelat's other books remain untranslated until today.

What is called now "Community of Scholars" at the Princeton Institute for Advanced Study (PIAS) then seems to have been a real in-group. It included W. Pauli who had spent the summer term of 1954 there with previous stays in 1935-1936, 1940-1946, and 1949-1950; V. Bargmann had spent terms at PIAS in 1938-1946 and 1954-1955; M. Fierz stayed there during the fall term of 1950, Oskar Klein spent a term at PIAS in 1949-1950 giving atomic physics and relativity theory as his interests. Neither someone from the Hamburg group (Jordan, Ludwig, Just) nor from the French groups (Lichnerowicz, Thiry, Tonnelat) has resided at the Institute for Advanced Studies in Princeton. Two scientists could have acted as go-between with regard to Paris and Princeton, Cécile DeWitt-Morette, at PIAS in 1948-1950, and Yvonne ChoquetBruhat in 1951-1952. But they had shown no special interest in gravity; DeWittMorette while working in theoretical physics was interested in quantum field theory;

\footnotetext{
37 Dieter Brill, professor at the University of Maryland.

38 Private communication of 8 April 2012 by D. Brill to the author.
} 
Choquet-Bruhat's focus was on partial differential equations. She stayed in Princeton in 1951/1952 and also in 1955 when her husband Gustave Choquet (1915-2006) was likewise in the Mathematics section at PIAS. Of course, André Weil has spent a sizeable part of his career there: from 1958 to 1976. Wether closer contacts between him and André Lichnerowicz have existed is unknown to me. Also, I have no information about the relations between Princeton University and the Sorbonne in Paris. According to D. Brill, it is likely that Lichnerowicz has come through Princeton during the late 1950 s to early 1960 s.

As to citations, Scherrer, Jordan, Thiry, and Jonsson could not have cited Brans and Dicke. The only symmetric citation was Jordan $\longleftrightarrow$ Thiry. We find a number of oneway citations, e.g., Jordan $\rightarrow$ Jonsson; Scherrer $\rightarrow$ Ludwig and Müller; Jonsson $\rightarrow$ Thiry; Dicke $\rightarrow$ Jordan; Dicke and Brans $\rightarrow$ Jordan; Brans $\rightarrow$ Jordan, Ludwig, Just. After the papers of Brans and Dicke had appeared, those in Paris still working on unified field theory took no notice of them. This refers to both, research on Jordan-Thiry theory [73], and on Einstein-Schrödinger theory [74]. Only M.-A. Tonnelat, in her books on the experimental verifications of general relativity (1964), and on the history of the relativity principle (1971), eventually cited both R. Dicke and C. Brans. While in the second book they are mentionend only in passing in connection with Mach's principle, [75, p. 516], in the first one, Tonnelat did not give much weight to BransDicke scalar-tensor theory: "Recently, Brans and Dicke [...] proposed a theoretical interpretation of the variation of constants. [...] Actually, to us this hypothesis seems to be too aleatory anyway to here devote to it important theoretical consequences" [76, p. 261]. ${ }^{39}$

As to secondary literature, I have found only three citations of the later papers by W. Scherrer [41,42] - all given after the emergence of Brans-Dicke theory, but none to his first note of 1941. In 1965, in a critique of a version of Hoyle-Narlikar gravitational theory, S. Deser and F. A. E. Pirani credited Scherrer for having obtained ("with a Machian motivation") similar wave equations [77, p. 143]. In another paper, they described Scherrer's (and Dicke's) theory as "in effect conventional versions of the Hoyle-Narlikar theory" [78, p. 449]. E. S. Harrison in 1972 gave a reference to Scherrer's paper of 1949 in a list of papers concerning scalar-tensor theory without any comment [79]. ${ }^{40}$

\section{Conclusions}

The previous discussion shows that the genesis of scalar-tensor theory is not just a story about different people arriving independently at the same theory: It is more intricate with some of the originators obtaining the same formal theory and others a

\footnotetext{
39 Récemment, Brans et Dicke [...] ont proposé une interprétation théorique de la variation des constantes. [...] Actuellement, cette hypothèse nous semble néanmoins trop aléatoire pour que des dévelopments théoriques importants lui sont ici spécialement consacrés.

${ }^{40}$ For macroscopic phenomena, the Hoyle-Narlikar theory referred to its equivalent to Einstein's theory. Cf. ([80], pp. 198-200). For the difference between Hoyle-Narlikar and Brans-Dicke theory, see also [81], pp. 196-200).
} 
different one. The physical interpretations, if available, also vary. During the process of theory-building, some of the researchers have come into contact with each other.

Now it is clear why Brans-Dicke theory made such an impact. It was the slightest alteration of general relativity of all scalar-tensor theories, and presented in a way that everyone familiar with Einstein's theory immediately could work with it without having to learn new techniques. No strange five-dimensional spaces around, no weird mass generation as a consequence as in Jordan's original interpretation! No connection to unified field theory which Thiry held high although it already carried the anathema of an influential physicist of the time as W. Pauli. Certainly, some of the physical ideas going into Brans-Dicke theory were either not new like the conception of a variable gravitational constant. Or, they were on shaky ground like the invocation of Mach's principle, ill defined for a field theory and still living a meager life on the fringes of physics. A new aspect with regard to Jordan was the discussion concerning the weak and strong equivalence principle; it was most important to Dicke because of his interest in devising and doing experiments on gravitation. Up to this day, a possible violation of the weak equivalence principle is the starting point for speculative theory building. Jordan had to look for geophysical applications (expansion of the Earth's crust) and to cosmology both of which were not open to local experimentation. No doubt, the Lagrange function and field equations, and the coupling with matter of Brans-Dicke theory had been published years before by others. But Brans and Dicke opted for the one Lagrangian, Fierz had shown to be irrefutable, and thus they cleared up the clutter caused by the indeterminacy in the papers of Jordan's coworkers Ludwig, Müller and Just.

Coming to external factors for the immediate acceptance of scalar-tensor theory à la Brans-Dicke: Princeton University with the impressive figure of J. A. Wheeler in the community of relativists and R. H. Dicke as a highly respected experimental and applied physicist [82-84], was certainly one of the best places for authors to launch an alternative theory of gravitation. Jordan's early papers and his books all were written in German knowledge of which was decreasing rapidly after the second world war. Also, some of his publications were hidden in special journals, perhaps unavailable in most American libraries; in any case they seemingly were not read abroad. At first, he had put into the foreground his research on projective relativity, and he remained unclear with regard to the coupling of the new scalar function to matter. ${ }^{41}$ As we have seen, interaction of the universities of Paris and the Institute for Advanced Study in Princeton in the field of mathematics had been notable at the time, but apparently there was very little exchange in terms of physics. Also, the French scientists involved in unitary field theory using Kaluza's five-dimensional theory, almost without exception published in French. ${ }^{42}$

Willy Scherrer, a former president of the Swiss Mathematical Society (1938-1939), was the first one who had proposed a scalar-tensor theory with formally the same

\footnotetext{
41 A further reason for reservation, in some circles in the United States, might have been Jordan's nationalistic attitude during the Nazi regime. This is mentioned by Carl Brans [6, p. 5]. Jordan's past during the Nazi regime is presented in [85].

42 Whether an influential figure like L. de Broglie with his negative stand toward quantum theory stood in the way of a stronger interaction between these two places is another question.
} 
Lagrangian Brans and Dicke were to use 20 years later. Nevertheless, he was ignored by the community of relativists-his Swiss colleagues and P. Jordan included. In his applied-mathematics-attitude with regard to scalar-tensor theory, he may be compared to Y. Thiry, inclined more toward mathematical physics. Apparently, Scherrer was not much respected by W. Pauli and M. Fierz and excluded from their communicationlines.

In comparison with the recent learned fights about the genesis of general relativity, no high stakes were connected with scalar-tensor theory. No battles about priorities ensued, no good anecdotes seem to have survived. Nevertheless, from this case study we may learn how intimately connected speculative, empirical and mathematical approaches to an alternative theory of gravitation have been. And, that communication between scientists in different countries was not as satisfactory as it might have been.

Acknowledgments My thanks go to Dieter Brill for communicating some of his memories of the time to me, to Friedrich Hehl for helpful comments and for a copy of Just's Habilitationsschrift, and to Norbert Straumann for allowing me to quote from Fierz's letter. S. Deser helped me to revalue his references to Scherrer and corrected a biographical mistake. A reference pointed out to me by a referee is also included.

Open Access This article is distributed under the terms of the Creative Commons Attribution License which permits any use, distribution, and reproduction in any medium, provided the original author(s) and the source are credited.

\section{References}

1. Kaiser, D.: When fields collide. Sci. Am. 296(6), 62-69 (2007)

2. Nordström, G.: Zur Theorie der Gravitation vom Standpunkt des Relativitätsprinzips. Ann. Phys. Leipzig 42, 533-554 (1913)

3. Coquereau, R., Esposito-Farese, G.: The theory of Kaluza-Klein-Jordan-Thiry revisited. Ann. Inst. H. Poincaré 52(2), 113-150 (1990)

4. Fujii, Y., Maeda, K.: The Scalar-Tensor Theory of Gravitation. University Press, Cambridge (2003)

5. Brans, C.H.: The roots of scalar-tensor theory: an approximate history. arXiv. org; arXiv:grqc/0506063v1. Accessed 3 April 2012

6. Brans, C.H.: Scalar-tensor theories of gravitation: some personal history. Contribution to Morella proceeding, p. 2. http://loyno.edu/ brans/ST-history/. Accessed 14 May 2012 (2008)

7. Dirac, P.A.M.: The cosmological constants. Nature 139(3512), 323 (1937)

8. Dirac, P.A.M.: A new basis for cosmology. Proc. R. Soc. Lond. A 165, 199-208 (1938)

9. Jordan, P.: Zur projektiven Relativitätstheorie. Nachr. Akad. Wiss. Göttingen. Math.-Phys. Kl. 39-41 (1945)

10. Jordan, P.: Relativistische Gravitationstheorie mit variabler Gravitationskonstante. Naturwiss. 11, 250-251 (1946)

11. Jordan, P., Müller, C.: Field equations with a variable 'constant' of gravitation. Z. Naturf 2a, 1-2 (1947)

12. Ludwig, G.: Der Zusammenhang zwischen den Variationsprinzipien der projektiven und der vierdimensionalen Relativitätstheorie. Z. Naturf. 2a, 3-5 (1947)

13. Bergmann, P.G.: Unified field theory with fifteen variables. Ann. Math. 49, 255-264 (1948)

14. Jordan, P.: Fünfdimensionale Kosmologie. Astr. Nachr. 276, 193-208 (1948)

15. Ludwig, G., Müller, C.: Ein Modell des Kosmos und der Sternentstehung. Ann. Phys. Leipzig 2(6), 76-84 (1948)

16. Pauli, W.: Wolfgang Pauli. Wissenschaftlicher Briefwechsel. In: von Meyenn, K (ed.), vol. III. Springer, Berlin, Heidelberg (1993)

17. Ludwig, G., Müller, C.: Ein Modell des Kosmos und der Sternentstehung. Arch. Math. 1(1), 80$82(1948)$

18. Ludwig, G.: Zur projektiven Relativitätstheorie mit variabler Gravitationskonstanten, 2. Mitteilung: Variationsprinzipien und Feldgleichungen für Gravitation und Materie. Z. Phys. 125, 545-562 
19. Bergmann, P.G.: Comments on the scalar-tensor theory. Int. J. Theor. Phys. 1, 25-36 (1968)

20. Wagoner, R.V.: Scalar-tensor theory and gravitational waves. Phys. Rev. D 1(12), 3209-3216 (1970)

21. Will, C.M.: Theory and Experiment in Gravitational Physics, Revised edn. University Press, Cambridge (1993)

22. Jordan, P.: Schwerkraft und Weltall. (Die Wissenschaft Bd. 133). 2. erweiterte Auflage. Bearbeitet unter Mitwirkung von E. Schücking. Braunschweig, Vieweg (1955)

23. Jordan, P.: Schwerkraft und Weltall. Grundlage der theoretischen Kosmologie. Braunschweig, Vieweg (1952)

24. Just, K.: Neue Feldgleichungen zur Jordanschen Gravitationstheorie. Z. Phys. 140, 485-493 (1955)

25. Ludwig, G., Just, K.: Zur Jordanschen Theorie der Materie-Entstehung. Z. Phys. 143, 472-476 (1955)

26. Just, K.: Erweiterte Gravitationstheorie und Periheldrehung. Z. Phys. 144, 411-427 (1956)

27. Just, K.: Zur Wahl von Feldgleichungen der projektiven Relativitätstheorie. Z. Phys. 139, 498503 (1954)

28. Just, K.: Zur Jordanschen Gravitations-Theorie. Habiltationsschrift, Freie Universität Berlin, 377 (1957)

29. Pauli, W.: Wolfgang Pauli Wissenschaftlicher Briefwechsel. In: von Meyenn, K. (ed.), vol. IV, part 2. Springer, Berlin, Heidelberg (1999)

30. Heckmann, O.: Besprechung Jordan, P. Schwerkraft und Weltall. Z. Astrophys. 40, 278-282

31. Ludwig, G.: Fortschritte der projektiven Relativitätstheorie. (Die Wissenschaft Bd. 105). Vieweg \& Sohn, Braunschweig (1951)

32. Fierz, M.: On the physical interpretation of P. Jordan's extended theory of gravitation. Helv. Phys. Acta 29, 128-134 (1956)

33. Lichnerowicz, A.: Les théories relativistes de la gravitation et de l'électromagnísme. Masson, Paris (1955)

34. Pauli, W.: Wolfgang Pauli Wissenschaftlicher Briefwechsel. In: von Meyenn, K. (ed.), vol. IV, part 3. Springer, Berlin, Heidelberg (2001)

35. Straumann, N.: Private communication to H. G., 16 April 2012

36. Pauli, W.: Die Relativitätstheorie. Reproduction of 1st edn. (1921) with supplements and additional comment. Boringhieri, Torino (1958)

37. Jordan, P.: Zum gegenwärtigen Stand der Diracschen kosmologischen Hypothesen. Z. Phys. 157, 112121 (1959)

38. Scherrer, W.: Zur Theorie der Elementarteilchen. Verh. Schweiz. Naturf. Ges. 121, $86-87$ (1941)

39. Scherrer, W.: Ein Ansatz für die Wechselwirkung von Elementarteilchen. Helv. Phys. Acta 14(1), 81$84(1941)$

40. Scherrer, W.: Bemerkungen zu meiner Arbeit: Ein Ansatz für die Wechselwirkung von Elementarteilchen. Helv. Phys. Acta 14(2), 130 (1941)

41. Scherrer, W.: Über den Einfluss des metrischen Feldes auf ein skalares Materiefeld. Helv. Phys. Acta 22, 537-551 (1949)

42. Scherrer, W.: Über den Einfluss des metrischen Feldes auf ein skalares Materiefeld (2. Mitteilung.). Helv. Phys. Acta 23, 547-555 (1949)

43. Heckmann, O., Jordan, P., Fricke, W.: Zur erweiterten Gravitationstheorie. I. Z. Astrophys. 28, 113149 (1951)

44. Fink, K.: Metrisches Feld und skalares Materiefeld. Com. Math. Helv. 25, 26-42 (1951)

45. Thiry, Y.: Étude mathématique des équations d'une théorie unitaire à quinze variables de champ. J. Math. pures et appl., Série 9, 30, 275-396 (1951)

46. Lichnerowicz, A., Thiry, Y.: Problèmes de calcul des variations liés à la dynamique classique et á la théorie unitaire du champ. Compt. Rend. Acad. Sci. Paris 224, 529-531 (1947)

47. Thiry, Y.: Les équations de la théorie unitaire de Kaluza. Compt. Rend. Acad. Sci. Paris 226, 216218 (1948)

48. Thiry, Y.: Sur la régularité des champs gravitationels et électromagnétiques dans les théories unitaires. Compt. Rend. Acad. Sci. Paris 226, 1881-1882 (1948)

49. Jonsson, C.V.: Studies on five-dimensional relativity theory. Ark. Fys. 3(8), 87-129 (1951)

50. Pauli, W.: Wolfgang Pauli. Wissenschaftlicher Briefwechsel. In: von Meyenn, K. (ed.), vol. IV, part 1. Springer, Berlin, Heidelberg (1996)

51. Einstein, A., Pauli, W.: On the non-existence of regular stationary solutions of relativistic field equations. Ann. Math. 44, 131-137 (1943)

52. Dicke, R.H.: Gravitation an enigma? Am. Sci. 47, 25-40 (1959) 
53. Dicke, R.H.: New research on old gravitation Science 129, 621-624 (1959)

54. Sciama, D.W.: On the origin of inertia Mon. Not. R. Astron. Soc. Lond. 113, 34-42 (1953)

55. Dicke, R.H.: Gravitation without a principle of equivalence Rev. Mod. Phys. 29, 363-376 (1957)

56. Brans, C.H., Dicke, R.H.: Mach's principle and a relativistic theory of gravitation Phys. Rev. 124, 925935 (1961)

57. Brans, C.H.: Mach's principle and a relativistic theory of gravitation. II Phys. Rev. 125, $2194-$ $2201(1962)$

58. Brans, C. H.: Mach's principle and a varying gravitational constant. Princeton dissertation, May 1961

59. Dicke, R.H.: Mach's principle and invariance under transformation of units Phys. Rev. 125, 21632167 (1962)

60. Dicke, R.H.: The earth and cosmology Science 138, 653-664 (1962)

61. Just, K.: Zur Kosmologie mit veränderlicher Gravitationszahl Z. Phys. 140, 648-655 (1955)

62. Just, K.: Zur Planetenbewegung bei veränderlicher Gravitationszahl Z. Phys. 140, 524-530 (1955)

63. Klein, O.: The atomicity of electricity as a quantum theory law Nature 118, 516 (1926)

64. Klein, O.: Quantentheorie und fünfdimensionale Relativitätstheorie Zeitschrift F. Physik 37, 895906 (1926)

65. Pauli, W.: Die Relativitätstheorie. Reproduktion der ersten Ausgabe v. 1921 nach der englischen u. italienischen Neuausgabe von 1958. Boringhieri, Torino (1963)

66. Klein, O.: Conference on New Theories in Physics, held in Kasimierz, Poland (1938). Cf. Helv. Phys. Acta 58(Suppl. IV) (1956)

67. Klein, O.: Meson fields and nuclear interaction oArkiv fri Matematik, Astronomi Och Fysik 34 A(1), $1-19$ (1947)

68. ORaifeartaigh, L., Straumann, N.: Early history of gauge theories and Kaluza-Klein theories, with a glance at recent developments. arXiv:hep-ph/9810524v2 (1999)

69. Espong, G.: The Oskar Klein Memorial lectures, vol. 1, pp. 85-102. World Scientific, Singapore (1991)

70. Einstein, A., Bergmann, P.: On a generalization of Kaluza's theory of electricity Ann. Math. 39, 683701 (1938)

71. Brill, D.: Review of Jordan's extended theory of gravitation. In: Moeller, C. (ed.) Proceedings of the International School of Physics "Enrico Fermi" vol. 20, pp. 50-68 (1962)

72. Tonnelat, M.-A.: La théorie du champ unifié d'Einstein et quelques-uns de ses développements. Gauthier-Villars, Paris (1955)

73. Surin, A.: Étude du schéma fluide parfait et des équations de mouvement dans les théories pentadimensioinelles de Jordan-Thiry et de Kaluza-Klein. Gauthier-Villars, Paris (1965)

74. Nguyen, P.C.: Contribution à l'etude des théories du champ unifié du type théorie d'EinsteinSchrödinger Ann. Inst. H. Poincaré 18(4), 303-357 (1964)

75. Tonnelat, M.-A.: Histoire du principe de relativité. Flammarion, Paris (1971)

76. Tonnelat, M.-A.: Les vérifications expérimnetales de la relativité générale. Masson et Cie, Paris (1964)

77. Deser, S., Pirani, P.A.E.: Critique of a new theory of gravitation Proc. R. Soc. Lond. A 282, 133145 (1965)

78. Deser, S., Pirani, P.A.E.: The sign of the gravitational force Ann. Phys. N. Y. 43, 436-451 (1967)

79. Harrison, E.R.: Scalar-tensor theory and general relativity Phys. Rev. D 6, 2077-2079 (1972)

80. Hoyle, F., Narlikar, J.V.: A new theory of gravitation Proc. R. Soc. Lond. A 282, 191-207 (1964)

81. Lord, E.A.: Tensors, Relativity, and Cosmology. Tata McGraw-Hill Publ. Co. Ltd., New Delhi (1976)

82. Dicke, R.H.: Eötvös experiment and gravitational redshift Am. J. Phys. 28, 344-347 (1960)

83. Dicke, R.H., Krotkov, R., Roll, P.G.: The equivalence of inertial and passive gravitational mass Ann. Phys. N. Y. 26(3), 442-517 (1964)

84. Dicke, R.H., Goldenberg, H.M.: Solar oblateness and general relativity Phys. Rev. Lett. 18, 313316 (1967)

85. Hoffmann, D., Walker, M.: Pascual Jordan (1902-1980) "Der gute Nazi-Pascual Jordan und das Dritte Reich”, pp. 83-111. Preprint 32983-111, Max Planck Institute for the History of Science, Berlin. http://www.mpiwg-berlin.mpg.de/Preprints/P329.PDF 